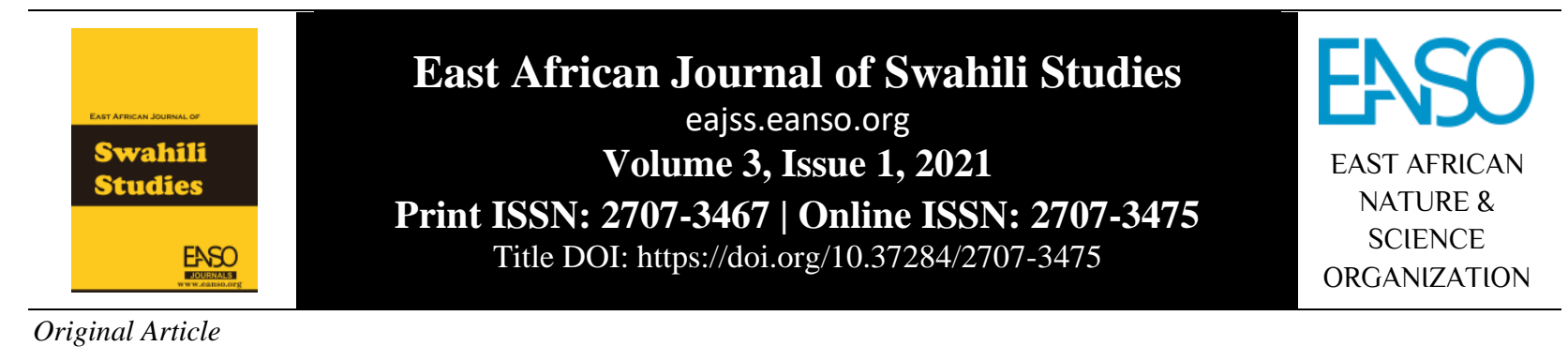

\title{
Jinsi Watoto Wanavyokabiliana na changamoto kwenye vitabu vya fasihi.
}

Viola Jepkemboi Kiplagat ${ }^{1}{ }^{*}$ Dkt. Rebecca Wanjiku Omolo, PhD ${ }^{1}$ na Margan Adero, PhD ${ }^{1}$

${ }^{1}$ Chuo kikuu cha Catholic, S.L.P. 4803, Eldoret, Kenya.

*Barua pepe ya mawasiliano: viokiplagat82@gmail.com.

DOI ya Nakala: https://doi.org/10.37284/eajss.3.1.405

Tarehe ya Uchapishaji: IKISIRI

08 Septemba 2021 Watoto wengi wanapitia changamoto nyingi miongoni mwa walezi wao na wanajamii. Kutokana na yale wanayoyapitia pamoja na mazingira duni

\section{Maneno Muhimu:}

Fasihi Ya Watoto,

Kukabiliana,

Migogoro,

Kutekeleza,

Mahitaji. kukosa mahitaji yao ya kuwawezesha kuwa na hulka zinazokubalika katika jamii. Mtafiti alinuia kuchanganua vitabu vya fasihi ya watoto ili kubainisha iwapo vitabu hivi vinaweza kutekeleza mahitaji ya watoto kupitia migogoro au changamoto vitabuni. Vitabu teule vilivyotumiwa katika utafiti vilikuwa Ahaa! Roda (2017) kilichoandikwa na Tom Nyambeka na Mwisho wa Ujambazi (2017) naye Yahya Mutuku. Nadharia iliyotumika katika utafiti huu ni nadharia ya Maadili na nadharia ya Uhalisia. Njia za utafiti zilizotumiwa ni hojaji na upekuzi wa yaliyomo kwenye vitabu teule vya fasihi ya watoto. Data iliyokusanywa ilipangwa kulingana na maswali ya utafiti na kuchanganuliwa kwa kuzingatia mkabala mseto ambapo, muundo wa QUAL-QUAN ulitumika. Mtafiti alichanganua vitabu teule vya fasihi kimaelezo akiongozwa na maswali ya utafiti. Mtafiti alikusanya data nyanjani kupitia hojaji zilizosambazwa kwa sampuli ya asilimia kumi ya watoto mia mbili kutoka darasa la saba, shule ya msingi ya Langas, Kaunti ya Uasin Gishu. Shule hii ya Langas ni shule iliyoko katika mazingira duni hivyo basi watoto wengi wanapitia changamoto nyingi za kijamii. Matokeo yalibainisha kuwa vitabu vya fasihi vinaweza kutekeleza mahitaji ya watoto kupitia migogoro vitabuni na kwamba vitabu vya fasihi ya watoto vinaweza kutoa mwelekeo kwa watoto kuhusu jinsi ya kukabiliana na changamoto katika hali halisi. Watoto 50\% waliotafitiwa walikubali kuwa vitabu walivyovisoma vilikuwa changamoto ambazo waliweza kufananisha na hali halisi. Changamoto kwenye vitabu teule zilioana na changamoto halisi za watoto. Utafiti huu ulitoa mapendekezo kwa taasisi ya KICD na wizara ya elimu watumie utafiti huu kufanya tafiti zaidi ili kuchangia zaidi mitaala kwa lengo la kutekeleza mahitaji ya watoto. Walezi, walimu, wakuu wa shule

108 | This work is licensed under a Creative Commons Attribution 4.0 International License. 
watumie utafiti huu kuwalea watoto wao kwa njia mbadala na wenye manufaa. Utafiti zaidi uweze kufanywa kuhusu fasihi ya watoto kutekeleza mahitaji ya watoto kupitia changamoto zinazotokana na migogoro vitabuni. Waandishi wa vitabu pia watumie utafiti huu kuandika vitabu vilivyo na maudhui tofauti tofauti yanayoguza changamoto za watoto na hali halisi ya watoto.

\section{APA CITATION}

Kiplagat, V. J., Omolo, R. W., \& Adero, M. (2021). Jinsi Watoto Wanavyokabiliana na changamoto kwenye vitabu vya fasihi. East African Journal of Swahili Studies, 3(1), 108-123. https://doi.org/10.37284/eajss.3.1.405

\section{CHICAGO CITATION}

Kiplagat, Viola Jepkemboi, Rebecca Wanjiku Omolo, and Margan Adero. 2021. "Jinsi Watoto Wanavyokabiliana na changamoto kwenye vitabu vya fasihi". East African Journal of Swahili Studies 3(1), 108-123. https://doi.org/10.37284/eajss.3.1.405.

\section{HARVARD CITATION}

Kiplagat, V. J., Omolo, R. W. and Adero, M. (2021) “Jinsi Watoto Wanavyokabiliana na changamoto kwenye vitabu vya fasihi”, East African Journal of Swahili Studies, 3(1), pp. 108-123. doi: 10.37284/eajss.3.1.405.

\section{IEEE CITATION}

V. J. Kiplagat, R. W. Omolo, and M. Adero, “Jinsi Watoto Wanavyokabiliana na changamoto kwenye vitabu vya fasihi”, EAJSS, vol. 3, no. 1, pp. 108-123, Sep. 2021.

\section{MLA CITATION}

Kiplagat, Viola Jepkemboi, Rebecca Wanjiku Omolo, and Margan Adero. "Jinsi Watoto Wanavyokabiliana na changamoto kwenye vitabu vya fasihi”. East African Journal of Swahili Studies, Vol. 3, no. 1, Sep 2021, pp. 108-123, doi:10.37284/eajss.3.1.405.

\section{UTANGULIZI}

Fasihi ya watoto ya Kiswahili inayoelezwa katika makala haya itatumika kwa maana ya fasihi iliyotungwa ama na watu wazima au watoto wenyewe, yenye kutumia lugha ya Kiswahili na kukusudiwa kusomwa au kutendwa na watoto (Bakize, 2013; Lyimo, 2014; Ngugi, 2015). Ingawa kwa ujumla fasihi hii ya watoto haitofautiani sana na ile ya watu wazima kwa dhana ya kutumia sanaa ya lugha.

Matundura, Kobia na Mukuthuria (2013) wanakubaliana kwa kina kwamba fasihi hii ina sifa kadhaa bainifu ambazo huitofautisha na kazi nyingine za watu wazima. Sifa hizo ni pamoja na kutumia lugha rahisi, kuwa na visa vichache, mambo mema kuwa ndiyo matarajio makuu, kutumia watoto kama wahusika wakuu, kuwa na msuko sahili, hadhira yake kuwa watoto wenyewe, maisha ya wahusika wakuu kuanzia na kuishia nyumbani, kutumia fantasia kwa kiasi kikubwa, miongoni mwa mengine.

Fasihi hii ya watoto ina tanzu kama za fasihi nyingine ya watu wazima. Mathalani, katika utanzu simulizi kuna hadithi, semi, nyimbo na katika utanzu andishi kuna hadithi fupi, riwaya, tamthilia na ushairi. Fasihi kama kioo cha jamii inaweza kuathiri sana wasomaji wake. Watoto wanakabiliwa na changamoto nyingi sana katika maisha yao ya kawaida. Wengi wanajitahidi kwa njia moja au nyingine kwa ajili ya kuishi katika mazingira yaliyo na changamoto si haba dhidi yao. Kulaumu picha za uharibifu wa maadili, unyanyasaji usio na maana, umasikini ulioenea, ujauzito na matumizi mabaya ya madawa ya kulevya kati ya watoto ni kile tunachokiona kila siku (Mapunjo, 2014).

Fasihi inaweza kutumika ili kuongeza uelewaji wa kibinafsi na kukuza utu wa watoto (Dada na Olaniyan 2020). Wao wanabainisha kuwa katika mchakato wa kusoma fasihi, mawazo ya wasomaji wengine wa ulimwengu yanasisitizwa tena, kugeuzwa, au hata yanakataliwa na hii inaweza kusababisha kugeuza msomaji. Hapa, ufanisi wa vitabu, usomaji na uaminifu wa maandishi ni muhimu kwa mchakato uliyoelezewa. Suala hili linafafanuliwa zaidi na Crippen (2012) kuwa anaelezea usomaji wa maandiko kama uzoefu mkubwa wa kibinafsi unaweza kusaidia kutimiza mahitaji ya watoto, kuongezeka kwa kuelewa 
wengine na kuweka kikamilifu zaidi ya maadili na kanuni.

Katika nchi za Ulaya, Martinez na wenzake (2018), wanaonyesha kwamba maandiko yanaweza kuwa silaha yenye nguvu zaidi ambayo inaweza kutumika kurekebisha mawazo ya watoto wengi. Wanasema kuwa kazi muhimu zaidi ya maandiko ni kusambaza maadili ya kitamaduni, maadili na tabia njema. Maadili haya wanayosema yanaweza kuendeleza picha ya watu au taifa.

Maandiko katika vitabu vya hadithi yanaweza kuwaongoza kupata ugunduzi wa kujitegemea, na yanaweza kuwasaidia kuondokana na maovu katika jamii. Maandishi pia yanaweza kuwasaidia kudhibiti vyema hisia za kibinafsi. Hii inaungwa mkono na maneno ya Lacina na Stetson (2013), kuwa fasihi ina jukumu la kuimarisha uundaji wa maadili na hisia za ushirikiano, usawa na sifa imara za uvumilivu, huruma, kugawana, kujali, wajibu wa kiraia na uwezo wa kutatua migogoro kupitia njia zisizo za kivita na msimamo muhimu.

Katika Afrika, dhana ya jinsia ni sehemu moja ya jamii ya kibinadamu inayoonekana katika vitabu vya watoto. Hivyo, mawazo kuhusu mahitaji ya watoto ya kawaida na kukubalika kwa wanaume na wanawake yanaweza kuimarishwa kupitia vitabu hivi (Heather \& Carmen 2011). Kwa hiyo watoto mara nyingi huiga mifano ya tabia kwa urahisi ambayo inapatikana kutolewa katika vitabu ambavyo wanavisoma. Mara nyingi, hasa katika vitabu vya watoto wa Afrika, mifano hii ya tabia huigwa.

Nchini Kenya, Odaga (1974) katika utafiti wake wa vitabu vya watoto na vijana katika jamii ya kabla ya ukoloni, anasema kuwa vitabu vilikuwa vinathaminiwa kama mojawapo ya njia kuu ambazo jamii ilielimishwa, kufundishwa na kuhusishwa watoto na vijana wake. Alihisi kuwa hii ilikuwa njia nzuri sana ya kuwashirikisha watoto na vijana.

Adungo katika gazeti la Taifa Leo tarehe 17 Machi 2020, anakubali kuwa fasihi ya watoto imepiga hatua kiasi fulani japo katika maeneo mengi bado ni changa mno. Fasihi ya watoto inayofahamika katika jamii nyingi za Afrika ya Mashariki, kwa kiasi kikubwa ni ile iliyo katika baadhi ya vitabu vya hadithi (ngano) na nyimbo za kitoto za makabila mbalimbali. Watoto wanapaswa kulelewa na kupewa vionjo mbalimbali vya malezi na elimu kupitia fasihi yao.

Vitabu vya fasihi vinaweza kutumika kama chombo cha kutimiza mahitaji ya watoto, kuwasaidia watoto kupata ujuzi na maarifa wanayohitaji kuimarisha na kutangamana na jamii. Waandishi wa vitabu vya fasihi ya watoto huzingatia matatizo ya kisasa zinazoonyesha changamoto za watoto wanapokuwa watu wazima katika jamii ngumu. Aina hii ya fasihi inaweza kutumika kukuza wajibu wa vitendo na hisia. Hii ni rasilimali ambayo inaweza kuandikwa ili kuokoa watoto nchini Kenya. Kwa hivyo, utafiti huu ulinuia kuchunguza iwapo vitabu vya fasihi ya watoto vilikuwa na uwezo wa kutimiza au kutekeleza mahitaji ya watoto ndani ya mchakato wa usomaji wa vitabu hivi.

\section{SUALA LA UTAFITI}

Matatizo yanayowakumba watoto katika jamii ni mengi. Haya ni kulingana na wataalamu kama vile Mapunjo, (2014). Matatizo hayo ni kama vile, umaskini, picha za uharibifu wa maadili, ujauzito, unyanyasaji na matumizi mabaya ya madawa za kulevya. LHRC, (Legal And Human Right Center, (2017) katika siku ya kuadhimisha siku ya mtoto wa Afrika iliangazia matatizo yaliyowakumba watoto. Matatizo hayo yalikuwa ubakaji na ulawiti wa watoto, ndoa za utoto chini ya miaka 18 , mimba za utotoni mashuleni, vitendo vya kikatili na mateso dhidi ya watoto, ukeketaji, vikwazo katika kutekeleza sera za elimu bure na huduma hafifu za afya.

Gazeti la Taifa Leo (2020) tarehe 17 mwezi wa 3 liliangazia ongezeko la mimba za mapema fumbo linalotatiza wataalamu. Tatizo hili linasababishwa na unajisi, ukosefu wa masomo na nafasi duni za kazi, ukosefu wa usawa kijinsia, kuzembea kwa wazazi katika malezi, dhuluma za kijinsia. Utafiti wa Hazina ya Umoja wa Mataifa unaohusika na idadi ya watu UNFPA (United Nations Population Fund) unaonyesha kuwa, kati ya Juni 2016 na Julai 2017 wasichana 378,397 katika umri wa miaka 10 hadi 19 nchini Kenya walishika mimba. Wasilwa (2021) alitoa ripoti ya Kenya NACADA (National Authority for the Campaign Against Drug Abuse), iliyosema kuwa watoto wadogo wenye umri wa miaka minne wanatumia dawa za kulevya. Ripoti hiyo ilionyesha kuwa takriban asilimia 7.8 ya 
watoto katika shule za upili wametumia dawa za kulevya. Ni wazi kuwa kuna matatizo mengi yanayowakumba watoto katika jamii hivyo mtafiti alijaribu kuona kama vitabu vya fasihi ya watoto vyaweza kutekeleza mahitaji ya watoto ili kuwaepusha kutokana na uozo wa jamii.

\section{UHAKIKI WA MAANDISHI}

Ili kuweza kukabiliana na changamoto za watoto, ni muhimu kutambua kuwa changamoto za watoto vitabuni husababishwa na kuwepo kwa migogoro vitabuni (Krebs, 2020). Mtafiti alitanguliza kwa kuangazia kwa upana yaliyoandikwa kuhusu migogoro kisha baadaye kuangazia yaliyoandikwa kuhusu ukabilianaji wa changamoto vitabuni.

\section{Migogoro.}

Huko Ulaya, kuna kazi nyingi zinazotolewa kuhusu migogoro. Kulingana na Lukov (2019) asili ya neno mgogoro imeshikamana na neno la Kilatini conflictus mgongano-mgongano, mgomo, kupigana. Kulingana na kamusi elezo huru, Migogoro ni mivutano na misuguano mbalimbali katika kazi za fasihi. Migogoro inaweza kuwa kati ya wahusika, familia zao, matabaka yao, au hata katika nyadhifa mbalimbali, ambayo hujitokeza katika mitazamo tofauti kulingana na mtiririko wa visa na matukio yanavyopangwa na mwandishi.

Kuna aina tofauti za migogoro, ambayo huunda msingi wa mgawanyiko wao, huweka kanuni tofauti. Ni muhimu kuzingatia kwa undani zaidi maudhui ya mgogoro. Mapambano ya wahusika wakuu katika ufahamu wao, tofauti ya hali yao, hisia zao, mawazo na hata tamaa husababisha mgongano. Kwa lugha rahisi, hii ni mapambano kati ya mema na mabaya, upendo na chuki, ukweli na uongo. Tangu jadi maudhui ya migogoro imetumika sana na waandishi mbali mbali wa fasihi.

Dickens (2020), aliyeandika kitabu maarufu Oliver Twist kwa mfano anaonyesha mapambano ya mtoto kujitegemea kimaisha kutokana na hali duni ya kimaisha na umaskini. Blake (2020), anaonyesha mambo yanayosababisha mgawanyiko wa vizazi. Mtaalamu huyu alitaja baadhi ya mambo yanayosababisha mgogoro kati ya watoto na wazazi. Kwanza, ni uhusiano wa kimapenzi, dini, Kelele au sauti ya juu, matumizi ya simu, alama wanazopata shuleni, usawa, mavazi, usawa, matumizi ya madawa za kulevya, matumizi mabaya ya raslimali kama vile stima na maji. Anaonyesha migogoro kati ya wazazi na watoto wao.

Thompson, Goodman na Waters (2018), wanaeleza kuwa mgogoro kati ya wazazi na watoto huibuka wakati wazazi wanapojaribu kuwaelekeza watoto wao kitabia. Watoto hawa huwa hawapendi kuelekezwa kwa kila jambo. Wanaendelea kusema kuwa, si lazima migogoro yasababishwe na mambo hasi bali hata mambo chanya. Wanasisitiza kuwa, migogoro huathiriwa na umri wa mtoto. Migogoro yanayoibuka kati ya mtoto chini ya miaka mitano hutofautiana na migogoro yanayoibuka kati ya mtoto aliyefika umri wa kubaleghe na mzazi wake.

Mgongano na ugomvi tunayopata katika kila kipande cha sanaa, iwe ni hadithi fupi, riwaya ya kihistoria au drama, kuwepo kwa mgogoro kunaweza kuleta mwelekeo wa kiitikadi. Porter (2008) katika kitabu chake cha The Cambrigde Introduction to Narratives anasema kuwa kunaweza kuwa na aina nyingi za migogoro katika hadithi moja, iwapo wahusika watakuwa na hamu zaidi ya moja au watapambana na hali zaidi ya moja. Mgogoro ndani ya mtoto mwenyewe au binafsi ni mojawapo ya aina ya migogoro katika fasihi. Haya ni maswala ya ndani ambayo huathiri matendo yao, motisha na maingiliano na wahusika wengine.

Porter (2008) anaendelea kusema kwamba migogoro kati ya watoto na wazazi ni aina nyingine ya migogoro. Katika hali nyingi kwenye jamii yetu, tunawasikia wazee wakilalamika jinsi vijana wa leo wanavyofanya mambo yao. Wazee huwaita watoto wa siku hizi' na kuwa hawana heshima wala adabu.

Shuleni pia ni vivyo hivyo kwani wanafunzi na walimu huishi katika hali ya kuogopana na kutoaminiana hivyo basi kuzuka migogoro wanafunzi wakishukiwa na waalimu wao kuwa na njama za utundu. Nao wanafunzi huona kuwa walimu wana haja ya kuwanyima haki na kuwakazia' kama wanavyosema vijana. Hali hii iliishia kuwa chanzo kikuu cha migomo na uchomaji wa shule nyingi nchini. Je, kunao uwezekano kwa wazee na vijana kuishi kwa ushirikiano na kuthaminiana katika tofauti‘ zao? Haya yaliungwa mkono katika makala ya Rokkit (2019).

111 This work is licensed under a Creative Commons Attribution 4.0 International License. 
Kwamba vizazi vishirikiane vyema na kutunzana ili kuepuka migogoro isiyokuwa na maana yoyote. Migogoro hii huwa haileti maendeleo yoyote bali huleta chuki na lawama zisizotatua chochote! Pengine suluhisho linaweza kuanzia maktabani ambapo watoto watajaribu kuvisoma vitabu vya fasihi na kujifundisha jinsi ya kutatua migogoro hiyo. Haya yalichangiwa na mwandishi aliyeandika kwenye Gazeti la Taifa Leo, Novemba 21,2016.

Aina ya tatu ya migogoro ni migogoro kati ya mtoto na jamii. Watoto haswa wanaweza kuwa na imani thabiti kinyume na kanuni za jamii. Inaweza kuwa maovu ya kijamii au ubaguzi unaoendelea katika jamii nzima inayo waathiri wachache kama vile ukabila, ubaguzi wa walemavu,watoto wa kike, waathiriwa wa ugonjwa hatari wa ukimwi ,uharibifu wa mazingira na mengine. The Alliance (2020) inayojumlisha UNICEF (United Nations International Children Emergency Fund) na WHO (World Health Organization) imetoa ripoti kuwa ipo migogoro kati ya watoto na jamii haswa wakati huu wa changamoto za covid-19. Watoto wengi wanapuuzwa na kunyimwa haki zao kutokana na umaskini unaokithiri. Ripoti hii imeonyesha kuwa kuna ongezeko la ukatili na unyanyasaji wa watoto. Watoto wamenyimwa uhusiano mzuri wa kutegemeana katika jamii.

Mtoto pia anaweza kupambana na migogoro kati yake binafsi na nguvu zisizo za kawaida kama vile vizuka, Mungu na miungu. Mambo haya yanazidi ufahamu wa kisayansi. Mazingira yaliyo na mambo kama haya kulingana na Porter (2008) huongeza mvuto kwenye hadithi. Kwa kuangazia migogoro kati ya watoto katika vitabu, tunaweza kutambua uhusiano wa migogoro hiyo na maisha yao ya kawaida. Pia watoto wanaweza kujitambulisha na wahusika vitabuni kupitia migogoro wanayokumbana nayo hivyo basi kupata mwelekeo chanya katika tabia zao.

Wanasaikolojia wengi wanakubaliana kuwa kuelewa mahitaji ya kimsingi ya watoto ni hatua muhimu katika kufanikisha maisha yao kitabia. Kwa mfano, Salehi (2011) anaeleza kuwa watoto wote huwa na mahitaji; na kwamba hisia za watoto huwa dhaifu kiasi kwamba mahitaji haya yakipuuzwa basi watoto wataweza kukabiliana na matatizo mengi katika siku za mbeleni. Hivyo basi, ili kupata mafanikio katika maisha ya watoto ya baadaye, ni muhimu kutambua mahitaji yao ya kimsingi, pawe na jitihada za kusuluhisha matatizo fulani wanayokumbana nayo na suluhu zinazotolewa zinahitaji kuwa zenye mantiki na zilizosawazishwa. Mahitaji haya yanaweza kutambulika kutokana na migogoro inayoibuka kati ya watoto.

Watoto huwa na mahitaji tofauti na wao hudhihirisha kuwa wanawategemea wazazi kuwatekelezea mahitaji haya. Mahitaji haya ni kama vile lishe bora, matibabu, elimu, mavazi, udadisi na usalama. Watoto huhitaji kudadisi na kutekeleza udadisi huo. Wao hutaka kudadisi mazingira yao kiuhalisi na kisaikolojia. Kwa sababu hiyo ni muhimu kufikiria kuhusu mada zinazofaa kwa kila mtoto katika vitabu vya watoto. Jambo hili litachangia katika kutimiza hamu yao ya udadisi. Ngugi (2014) anaeleza kuwa, ili mtoto aweze kufaidika kutokana na kile anachokisoma, ujumbe wa hadithi husika unahitaji kumhusu mtoto huyo. Hivyo basi migogoro itakayoangaziwa na mwandishi katika fasihi ya watoto inapaswa kutekeleza mahitaji haya.

Hivyo basi, utafiti huu ulinuia kuangazia migogoro katika vitabu teule kwa lengo la kuonyesha changamoto ambazo watoto wanapitia katika hali halisi. Waandishi wa vitabu vya hadithi za watoto wanapoangazia migogoro au changamoto ambazo watoto wanapitia katika uhalisia, watoto wanaweza kupata mielekeo chanya, misimamo, tabia na hata kuboresha mazingira yao kupitia vitabu hivi vya fasihi ya watoto.

\section{Changamoto za Watoto Kwenye Fasihi ya Watoto.}

Wanajamii wengi wamekuwa wakiinasibisha fasihi hii na mambo ya kitoto ambayo ni rahisi, yasiyo ya msingi sana; na ndiyo maana inaitwa fasihi ya watoto (Wamitila, 2008). Tafiti zinaonyesha kwamba fasihi hii imechipuka barani Afrika hivi karibuni, hasa kwenye miaka ya 1980 kutokana na sababu kadhaa lakini mojawapo ikiwa ni kutiliwa mkazo kwa masuala ya haki za watoto (Ngugi, 2015). Mulokozi (katika Semzaba, 2008) anaeleza kwamba nchini Tanzania, fasihi ya watoto ilichipuka wakati na baada ya ukoloni katika miaka ya 1960 na kushika mizizi miaka ya 1970. 
Katika utafiti uliofanywa na Ngugi (2011), walimu walioonekana kuwa na mielekeo chanya kwa vitabu vya ziada, baadhi wakidai kutumia mikakati mbalimbali kama njia ya kuwahimiza watoto kusoma. Baadhi ya mikakati hiyo ni kama vile; kuwapa watoto mazoezi ya kusoma, kuwa na majadiliano darasani kuhusu hadithi iliyosomwa, na kuwa na usomaji wa pamoja darasani. Usomaji wa vitabu vya fasihi katika shule za upili na uchambuzi wake.

Greenwood (1988) anapinga mbinu ya kuwapa watoto mazoezi baada ya kusoma kitabu chochote cha fasihi kwa, kudai kuwa mbinu hii hupunguza furaha na starehe ya kusoma. Katika hali kama hii, tunaweza kuwahimiza watoto kwa kuwaeleza kuwa, usomaji ni njia ya kujistarehesha na ili kupata starehe hiyo, ni muhimu kwa mwanafunzi kujibu maswali ili mwalimu ajue iwapo mwanafunzi ameielewa hadithi au la. Ni kutokana na sababu hii ambapo baadhi ya wachapishaji hujumuisha maswali ya ufahamu katika vitabu vya hadithi.

Hillman (1974) anaelezea kuwa waandishi hutumia lugha za kuunda picha za akili na halisi katika hadithi za watoto na hivyo kuwaelezea waandishi mtazamo wa dunia ambao una lengo la kushawishi na kuunda mtazamo wa wasomaji wao. Kulingana na yeye maandiko ni muhimu kwa akili, maendeleo ya kisaikolojia na kijamii ya mtoto. Vitabu vinasisimua mawazo ya watoto na huimarisha ufahamu wao juu ya ulimwengu unaowazunguka. Hizi ni kazi za uigizaji muhimu ambazo hufanya maandiko kuwa chombo muhimu katika mchakato wa kijamii.

Bakize (2013) anabainisha kwamba; ufundishaji wa fasihi ya watoto unaonekana kuachwa nyuma nchini Tanzania. Ili kazi za watoto ziwe nyingi, mitaala inapaswa kuijali fasihi hii ya kundi maalumu kwa sababu inaonekana wazi kuwa kundi hili la wanajamii bado halijapewa kipaumbele kifasihi kwa mkabala wa sera za elimu yetu. Pamoja na hili, Wamitila (2008) anasema kwamba, Pamekuwako na pengo kubwa katika ufundishaji wafasihi ya watoto na vijana katika taasisi zetu za kielimu kuanzia shule za sekondari hadi vyuo vikuu. Mitaala mingi inaelekea kutilia mkazo kwenye fasihi ya watu wazima. Pamoja nakwamba Wamitila alitoa kauli hiyo akirejelea hali ya mitalaa ya elimu nchini Kenya, hataTanzania hali ni hiyo hiyo.
Fasihi ya watoto ni ile iliyoandikwa kwa minajili ya kusomwa na watoto. Hii haimaanishi kuwa fasihi hii haiwezi kusomwa na watu wazima. Fasihi ya watoto huwa imeandikwa kwa kuzingatia msomaji ambaye hasa tajriba yake ya maisha ingali finyu na vilevile uelewa wake wa mambo ungali unakua. Hivyo basi, fasihi hii huwalenga watoto kwa kuwa wao ni binadamu ambao wanaendelea kukua kiakili, kilugha na hata kiumbo (Ngugi, 2014).

Fasihi huelimisha kwa kupanua mawazo ya watoto kutokana na maisha ya watu wengine. Mtoto anaposoma kazi yoyote ile, huwa anajielewa na kuuelewa ulimwengu anamoishi kupitia matukio katika kazi hiyo. Vilevile, kazi hiyo humsaidia kutalii ulimwengu wake, kukuza tajriba na kumpa nguvu za kuweza kukabiliana na hali mbalimbali zinazoweza kumjia. Katika ujumla wake, fasihi hutoa maonyo, mawaidha, makatazo na maelekezo. Hivyo basi, fasihi ya watoto ni njia mwafaka ya kuchuja jamii ya mtoto na kuielekeza. Katika fasihi ya watoto, msanii hujaribu kujenga ulimwengu mzima ambao unafanana na maisha halisi ambayo mtoto anaweza kujinasibisha nao. (Njogu na Chimera, 1999)

Katika ulimwengu wa sasa, watoto wamejikuta katika hali tatanishi ambapo wanahitaji ushauri kuhusiana na masuala ibuka katika jamii. Ngugi (2014) anaeleza kuwa watoto bado wanaendelea kukua kimwili, kisaikolojia na wanawategemea watu wazima kuwaelekeza. Mbali na ushauri wa moja kwa moja kutoka kwa wazazi, fasihi inaweza kuchangia katika kumsaidia mtoto kukubali hali fulani na hata kujua namna ya kukabiliana na hali nyinginezo ambazo zitaendelea kuibuka katika maisha yake kila kukicha. Matumizi ya fasihi kama chombo cha kutolea ushauri hukuza tabia inayokubalika katika jamii hivyo basi kukabiliana na changamoto wanazokumbana nazo watoto wanapoendelea kukua. Usomaji wa vitabu vya fasihi unaweza kutumika kama njia mbadala ya kuwatuliza watoto wanaopitia katika hali mbalimbali za kimaisha.

Kinyume na Greenwood (1988), mtafiti anaunga mkono wazo la kuwapa watoto maswali baada ya kusoma hadithi kwenye vitabu. Walezi pamoja na walimu wanaweza kutambua iwapo watoto wanasoma na kuelewa na jambo la muhimu zaidi linalohusiana na utafiti huu ni iwapo watoto 
wanapata mafunzo kutokana na yale wanayoyasoma kwenye vitabu vya fasihi hivyo basi mahitaji yao yakashughulikiwa.

\section{NADHARIA ZA UTAFITI}

Utafiti huu ulitumia nadharia mbili kwa pamoja ili ziweze kukamilishana na kuwezesha utafiti huu kutimiza malengo yake: Nadharia ya Maadili na Nadharia ya Uhalisia. Nadharia hutekeleza wajibu muhimu wa kutaalumisha uchanganuzi wa data ya fasihi simulizi. Nadharia ni mwongozo wa kuelekeza uchambuzi au uhakiki wa kazi za fasihi (Wanjala, 2020).

\section{Nadharia ya Kimaadili}

Kulingana na Oxford University Press Encyclopedia (2019), nadharia ya maadili hunuia kuchunguza matokeo yanayotokana na ukabilianaji wa yale yaliyoandikwa kwenye vitabu vya fasihi ya watoto. Nadharia ya maadili huchunguza changamoto na matokeo yaliyopo katika mazingira ya mtu binafsi na mambo ambayo ameweza kujifunza kutokana na uhusiano wake na mazingira hayo. Danley na wengine (2014) wanasema kuwa wakati mtoto anapojifunza mabadiliko yanapaswa kuonekana na kupimika.

Kendra (2019) anasema kuwa nadharia ya maadili pia inajulikana kama saikolojia ya tabia. Ni nadharia ya kuelimisha inayotegemea wazo kuwa mahitaji ya mtoto hupatikana kupitia hali fulani. Hali hutokea kutokana na mwingiliano na mazingira. Waasisi wa nadharia hii wanaamini kuwa majibu kutokana na kichocheo cha mazingira yanachagiza matendo au mahitaji ya mtoto. Kulingana na shule hii ya mawazo, mahitaji ya watoto yanaweza kufanyiwa utafiti kwa utaratibu na kwa namna ya pekee bila kujali hali ya akili. Kulingana na mtazamo huu, mahitaji ya watoto yanayoweza kuonekana huzingatiwa. Hisia na mhemko ni mifano ya mambo yasiyozingatiwa.

Kazdin (2001) anazungumza kuhusu Watson aliyekuwa mwanzishi wa kwanza wa Nadharia ya Maadili. Alitambua kuwa Watson alisisitiza kuwa viumbe vilivyo na uhai vilikuwa na uwezo wa kuzoea na kujihusisha na mazingira fulani. Alinukuu maneno ya Watson yafuatavyo, "Nipe watoto kadhaa wenye afya, walioumbwa vizuri, niwaweke na kuwalea katika ulimwengu wangu maalum na nitahakikisha kwamba nimemchukua kila mmoja kinasibu na kutoa mafunzo ya utaalamu mbali mbali kama vile daktari, mhandisi, mwanasheria, mkuu wa kijiji, mwanabiashara, mwizi na kadhalika bila kuzingatia vipaji vyao, uwezo, mielekeo, wito au hata ukoo". Haya ni kwa mujibu wa Kendra (2019). Watson alichunguza jinsi watu na baadhi ya wanyama walitoa majibu kufuatia msukumo wa aina fulani. Nadharia hii ilitoa mwongozo katika utafiti huu kwa kuzingatia kuwepo kwa vitabu vya fasihi ya watoto kama kichocheo cha hali ili kufanikisha lengo la wahusika katika kutekeleza mahitaji ya watoto. Mihimili ya nadharia hii ambayo itatuongoza katika utafiti huu ni: Fasihi ni zao la jamii, Fasihi hutoa mifano ifaayo ya maadili kwa wakati maalum, Mazingira ya mtunzi kijamii na kihistoria huathiri kazi yake na mwisho, Fasihi ni kielelezo cha maadili ya jamii kwa wakati maalum na mtunzi lazima azingatie maadili ya jamii yake kwa wakati wake ili kazi yake iwe faafu kwa jamii yake.

Maoni ya Wamitila (2003) ni kwamba kazi nyingi za kifasihi za Kiswahili zinaelekea kuegemezwa kwenye msingi wa kimaadili. Tunakubaliana naye kwa maoni hayo kwa sababu kuna kazi nyingi sana za kifasihi ambazo hujaribu kuwaelekeza wasomaji kuepukana na maovu katika jamii.

\section{Nadharia ya Uhalisia}

Nadharia ya uhalisia wa kijamaa katika fasihi huhitaji msanii kutupilia mbali mambo ya kubuni na badala yake kuwaza. Wanauhalisia wanasisitiza zaidi ukweli wa mambo kwa mujibu wa muktadha , historia na jamii husika. Nadharia ya Uhalisia ilihakikiwa na Defoe, Richardson, Fielding, Dickens na Balzac. Waasisi hawa wanasisitiza kuwa, ili fasihi iwe na manufaa katika jamii, yapaswa kuwa chombo cha kuichambua, kuikosoa, kuishauri na kuiokoa jamii. Uhalisia husisitiza kueleza na kueneza mawazo sahihi na kujaribu kutoa makosa katika jamii.

Lengo kuu katika uhalisia ni kuwafungua watu macho na kujaribu kutoa uchafu katika jamii (Senkoro, 1987). Mihimili katika nadharia ya Uhalisia ni kama ifuatavyo: Kwanza, kuzingatia kwa hali ya juu uhalisia katika kuwakilisha maisha kupitia sanaa ya fasihi. Pili, kuonesha mkabala wa wasomi, waandishi wa fasihi, falsafa na siasa. Tatu, 
ni sifa ya mtunzi wa fasihi au sanaa, na mwisho ni kiwango cha mtunzi kuwasilisha maisha jinsi yalivyo kwa uhuru au demokrasia.

Thamani ya kazi ya uhalisia wa kijamaa ni kuwa kielelezo kamili cha kazi ya fasihi. Kazi ya fasihi inapaswa kuchunguzwa ili kubainisha kuzorota kwa ujamaa. Nadharia ya Uhalisia huzingatia mambo yafuatayo; Uhusiano wa sanaa na nyakati tatu: wakati uliopo, ujao na uliopita. Pia kudhihirisha mahusiano katika jamii, jukumu la sanaa katika jamii lifahamike wazi na dhima ya msanii katika jamii aliyomo ibainike wazi (Wanjala, 2011). Nadharia hii ilitumiwa kuiweka fasihi ya watoto katika uhalisia.

\section{MUUNDO WA UTAFITI}

Muundo uliotumiwa ni wa mkabala mseto. Mkabala huu unatokana na mchanganyiko wa mbinu mbili yaani ya kitakwimu na ya kithamano. Mbinu hizi mbili zikitumika kwa pamoja hutoa matokeo ya kuaminika (Creswell 2008). Watafiti hutumia matokeo ya mbinu moja kuipa maelezo mbinu nyingine (Greene na wengine, 1989) ili kupata matokeo yaliyoaminika. Muundo wa mbinu fuatishi chini ya mkabala mseto ulitumika nayo ni mbinu ya QUAL-QUAN. Data za kithamano huunda data za kitakwimu katika mchakato wa ukusanyaji wa habari au maelezo (Creswell na Clark, 2007).

\section{Njia za Kukusanya Data}

Njia ni mbinu ambazo mtafiti anazitumia kutafuta habari muhimu kuhusiana na anwani. Hojaji, uchanganuzi wa matini na upekuzi wa nyaraka au maktaba (Wanjala 2020) zilitumika kukusanya data. Mtafiti alikusanya data kwa njia ya hojaji na upekuzi wa matini kwenye vitabu teule. Hojaji ilitumika kukusanya data kutoka kwa watoto wa darasa la saba. Vitabu vilivyopekuliwa ni viwili navyo ni Ahaa! Roda na Mwisho Wa Ujambazi.

\section{Mahali pa Utafiti}

Utafiti huu ulijikita nyanjani na kwenye maktaba. Nyanjani, Shule ya msingi ya Langas, kaunti ya Uasin Gishu, mkoa wa Rift Valley nchini Kenya, iliteuliwa kama mahali pa utafiti kwa sababu imo katika mazingira duni ambapo kila changamoto hupatikana. kama vile umaskini, utumiaji wa dawa za kulevya, ubakaji, uyatima, ngono za mapema, ujambazi, mauaji na mengine mengi (Kostelny, Wessels, Chabeda-Burthe na Ondoro 2013). Maktabani vitabu viwili vya fasihi ya watoto vilisomwa na kuhakikiwa na mtafiti. Hivi ni kifunzo cha Ahaa! Roda na Mwisho wa Ujambazi.

\section{Uwasilishaji wa Data.}

Uwasilishaji wa matokeo ulifanywa kwa njia ya maandishi ya maelezo na ufafanuzi. Uwasilishaji huu ulihusu maelezo kwa kurejelea vitabu vya fasihi ya watoto vilivyoteuliwa (Ahaa! Roda na Mwisho wa Ujambazi). Mtafiti pia aliwasilisha data akitumia jedwali, jedwali mduara na michoro.

\section{MATOKEO}

\section{Changamoto za Watoto Zinavyokabiliwa Kwenye Fasihi Ya Watoto}

Mtafiti alinuia kuonyesha jinsi vitabu vya fasihi ya watoto vinavyokabiliana na changamoto za watoto. Changamoto hizi zinajidhihirisha kupitia kwa migogoro inayoibuka kutokana na vitabu vya hadithi. Migogoro inapotatuliwa au suluhu ya migogoro inaonyesha suluhu ya changamoto za watoto. Hivyo basi, mtafiti alichanganua kwa upana migogoro iliyoibuka kati ya watoto katika vitabu viteule.

Mgogoro ni hali ya kutoelewana au kutoafikiana baina ya pande mbili. Mtafiti alionyesha na kueleza mifano ya migogoro inayopatikana katika vitabu vya fasihi ya watoto teule, Ahaa! Roda na Mwisho wa Ujambazi. Migogoro huendesha kazi nyingi za fasihi. Migogoro inaweza kuwa mapambano, kupigana, migomo, mgongano, mgawanyiko na misuguano. Hii inaweza kusababishwa na mapambano ya wahusika wakuu katika ufahamu wao, tofauti ya hali yao, hisia, mawazo na hata tamaa. Kuna aina za migogoro kama vile mgogoro ndani ya mtoto binafsi, migogoro kati ya watoto na wengine, migogoro kati ya watoto na mazingira na migogoro kati yao na nguvu zisizo za kawaida.

Migogoro katika maisha ya watoto ni muhimu kwani tunaweza kutambua mahitaji ya watoto na changamoto ambazo watoto wanapitia kibinafsi. Migogoro huweza kuleta mwelekeo wa kimaadili. Mtoto anapokutana na migogoro vitabuni, anaweza kujifunza kudhibiti hisia mbalimbali, anaweza kubadilisha mwenendo fulani au hata kupata ujuzi 
wa jambo fulani. Aina ya migogoro ilidhihirika kwenye vitabu hivi viwili. Kwanza, mtafiti aliangazia migogoro kwenye kitabu cha Ahaa! Roda kisha migogoro kwenye Mwisho wa Ujambazi.

Katika kitabu cha Ahaa! Roda kuna changamoto kuu ambayo ni kutoelewana kwa watu wa familia moja au mgogoro kati ya watu wa familia moja. Hii inajidhihirisha kupitia wahusika Sumu na marafiki wake ambao wanawateka nyara Roda na mamake (uk.8-13). Kitendo hiki cha kusikitisha kinafanyika kwa sababu ya mgogoro wa shamba ulioko kati ya Bi. Fatu ambaye ni mamake Roda na Sumu, ami wa Roda. Tamaa na wivu ulizua mgogoro huu Sumu alipoona maendeleo katika shamba la Bi. Fatu. Sumu alikuwa ameuza shamba lake lote kwa sababu ya ulevi. Sumu haoni haja ya mwanamke na mtoto msichana kuridhi shamba hivyo basi anapanga kuwaua. Mila na desturi imewadhulumu jinsia ya kike kuhusiana na uridhi wa mali. Changamoto hii haitatuki haraka. Inaishia kifo cha mamake Roda na Roda anakabiliana na hali ya uyatima uliyomfanya kuacha mali ya wazazi wake na kuenda kuishi na familia ya ami yake Kilemba. Mgogoro wa shamba uliisha baada ya kifo cha Bi. Fatu kwani Sumu na marafiki wake walishikwa na kupelekwa mahakamani. Walihukumiwa kifo mahakamani (uk.69).

Isitoshe, wanawake wanadhulumiwa mno kwa mfano, hadithi yaonyesha dhuluma wakati wanatekwa nyara na kutoridhishwa mali. Changamoto hii ilitatuliwa na Sajenti Kizito katika kitabu cha Ahaa! Roda alipotumia fursa aliyoipata baada ya Bi. Fatu kuaga kutetea haki za akina mama na watoto. Anawajulisha wananchi kuwa sheria zilimruhusu akina mama na watoto wa jinsia ya kike kuridhi mali. Alisisitiza kuwa utamaduni ulikuwa umepitwa na wakati (uk 52).

Mgogoro kati ya vizazi unaonekana pale ambapo Fadhili anaelezea ukali wa babake kutokana na matumizi ya Redio na umuhimu alioupata kutokana na kusikiliza Redio (Uk.29-31). Fadhili alipenda kusikiliza kipindi cha 'Ufasaha wa Lugha'. Kipindi hiki kilikuwa na mafunzo na makuzi mazuri ya lugha ya Kiswahili. Babake Fadhili anaona kuwa redio inaweza kuwapotosha watoto. Anawakataza watoto kusikiliza redio. Fadhili anaomba Roda redio yake na kusikiliza kipindi hicho kwa siri. Ingawa Fadhili alisikiliza redio kwa siri, alihakikisha kuwa babake hakuweza kumpata na kosa lile.

Kuna mgogoro kati ya wanakijiji ambao hawafurahii ushirikiano kati ya wanakijiji wengine na hawafurahishwi na ushirikiano kati ya familia hizi mbili ya Fadhili na Roda. Wazazi wa Fadhili pamoja na mamake Roda walipendana na kushirikiana katika shughuli za kilimo. Katika ulimwengu ambao umejaa gere, chuki na migao katika misingi ya kikabila, mgogoro hauepukiki pale ambapo wanakijiji wengi hawafurahishwi na ushirikiano huu wa familia hizi mbili (uk 6). Watoto wanapoona wazazi wao wakipitia mgogoro kama huu watafahamu umuhimu wa kushirikiana na kwamba watu wengi huwa hawafurahii mambo mema kwa wenzao.

Majanga anayopitia Roda yanazua mgogoro akilini mwa Fadhili ambaye ni fanani wa kitabu hiki. Kifo cha babake Roda, kutekwa nyara na kutishiwa maisha, hatari ya Sumu aliyetoroka mkono wa sheria kurudi na hata Roda kurudi nyuma masomoni (uk. 23) yanasababisha migogoro kali akilini mwa Fadhili. Anajaribu kadri awezavyo kumsaidia Roda kutokana na msongo wa akili na pia kufaulu masomoni. Fadhili hafi moyo anapambana na changamoto hizi na kujaribu kumsaidia Roda hadi anakuwa sawa. Fadhili anakabiliana na changamoto hizi kwa kuwa na subira na upendo wa dhati.

Mgogoro kati ya mtoto Roda na mazingira unaonekana pale ambapo mto wa Nena unaogawa kijiji cha Tupe na Mabatini kinafurika wakati wa msimu wa kifuku. Roda anasombwa na maji wakati mto ulipovunja kingo zake lakini anaokolewa na wapita njia. Mkoba ukiwa na vitabu pamoja na viatu vyake vilibebwa na maji. Roda anaendelea na masomo yake hata baada ya mkoba na vitabu kubebwa. Changamoto hii haimfanyi kuacha masomo.

Mgogoro kati ya mtu na mazingira unaendelezwa na Fadhili kwani ana maswali mengi kuhusu kifo na ardhi. Katika kijiji cha Tupe na Mabatini, visa vya mauaji, utekaji nyara na utapeli unaongezeka. Hali ya usalama unadorora. Bi. Fatu alipouawa, wanakijiji walijawa na hasira na simanzi. Fadhili anataja kuwa vifo vinakuwa vingi kiasi cha kutatiza masomo. Kati ya vifo zaidi ya kumi vilivyotokea, Fadhili alipoteza binamu yake kutokana na mhogo wenye sumu (uk 34). Fadhili ananong'onezana na 
mchanga ardhini. Kuna mabishano akilini mwake. Mchanga unamhakikishia kuwa alikuwa tayari kumpokea na kumhifadhi Bi. Fatu lakini Fadhili anajibu ardhi kuwa ilikuwa inammeza Bi. Fatu.

Kuna mgogoro akilini mwa Fadhili na ardhi kwani ardhi haikubaliani na maoni ya Fadhili na binadamu kwa jumla. Fadhili kwa hasira anamfokea ardhi kwamba ana tabia ya ukatili na ufidhuli. Ardhi inauwezo wa kufanya lolote ilitakalo (uk.48). Binadamu huwa hawakubaliani na kifo kwa lolote lile. Roda haelewi ni kwa nini mamake alimuacha mapema hivyo na alimuachia nani. Fadhili anagundua baadaye kuwa hakuwa na uwezo wowote juu ya ardhi. Fadhili anagundua pia kuwa hakuna kile atakachofanya ili kubadili hali.

Kuna mgogoro mkali kati ya Bi. Mnuna na Roda baada ya mamake kufa. Akilini mwa Bi. Mnuna kuna mgogoro usioeleweka iwapo alifurahia kuishi na Roda au la. Anaonekana mchovu wa mwili na akili kiasi cha kulazimisha tabasamu yake. Mumewe Bi. Mnuna anaporudi kazini, Mnuna anapata fursa ya kumtesa Roda vilivyo. Mateso na dhuluma alizopitia Roda mikononi mwa mke wa ami yake ni migogoro. Bw. Kilemba anapojulishwa kuhusu mateso ya Roda, anafika nyumbani kwa siri na wakiandamana na Bw. Tango. Mle ndani sauti za kukaripiana zinasikika. Mwishowe Bi. Mnuna anatoka chumbani upesiupesi mzigo kichwani. Watoto wanamfuata mama yao wakilia lakini wanarudishwa na baba yao. Changamoto hii inatatuliwa kwa kuondoka kwa mke wa Ami.

Mgogoro kati ya serikali na watoto wananchi unajidhihirisha Sajenti Kizito alimhakikishia Roda kwamba angepewa ufadhili wa aina yoyote, masomo, mavazi, lishe bora na kila hitaji la kimsingi mamake alipoaga (uk.52). Bw. Makali naye aliyefanya kazi katika kitengo cha ujasusi aliahidi kufuatilia maisha ya Roda. Kinyume na matarajio, Roda alidhulumiwa na kuteswa kiasi cha kuhofia masomo yake na hata kufa ilhali hakuna yeyote serikalini aliyefuatilia maisha ya Roda kama walivyoahidi. Roda alidhoofika kiafya, akakosa mavazi, usingizi, amani na lishe bora. Ami yake tu ndiye aliyemwokoa. Wazazi wanapoaga, watoto huachwa mayatima. Watu hujitokeza kabla ya mazishi kutoa ahadi ambazo hazitimiki. Roda anavumilia hata wakati serikali haimsaidii. Anavumilia hadi msaada wake unapopatikana.
Kitabu cha Mwisho wa ujambazi nacho hakijasazwa. Kina mifano ya migogoro chungu nzima. Kuna migogoro kati ya watu wafungwa au baina ya watu. Tabia za Matata zilizua migogoro ndani ya gereza kwa kuwadhulumu wafungwa wenzake. Aliwatendea visa na mikasa ya ajabu. Matata alipoachiliwa huru, wafungwa wengine walishangilia kwa hoihoi, vifijo na nderemo kwa sababu Matata alikuwa akiwadhulumu mumo humo gerezani (uk. 3). Kuna watu ambao tabia zao husababisha changamoto nyingi katika jamii. Ulevi, vita, uchoyo, tamaa, ufisadi ni baadhi tu ya mifano ya tabia hizi. Suluhu ya changamoto hizi ni watu kubadilika kitabia ili wakubalike katika jamii.

Mgogoro huu unaonekana pia katika basi ambalo Matata alipanda kwenda nyumbani. Kuna watu wengi katika kituo cha basi hivyo basi hali ya kuabiri basi haikuwa kawaida. Wasafiri walikimbilia mlango wa mbele na wengine wa nyuma. Baadhi yao walipita madirishani. Rabsha na fujo zilipamba moto huku akina mama wakiguna, ajuza wakibanwa na kutupa cheche za laana, mashaibu walilalama nao mabinti walipoteza fedha zao na wengine kuvunjiwa heshima (uk.4). Matata anamwibia mama kibeti kwa sababu ya tamaa na tabia yake ya wizi. Mama aliyeibiwa anaishiwa na stahamala na kuangua kilio kilichowavutia wasafiri (uk 5). Hakuna aliyetatua migogoro hii. Watu walivumulia changamoto hizi hadi mwisho wa safari. Ni muhimu watoto watambue kuwa suluhisho ya changamoto zingine huwa hazipo lakini watu wanaweza kutoa suluhisho zao kwa kubuni sheria zitakazofuatwa. Kwa mfano, sheria za barabarani zidhibiti viwango vya abiria kwenye matatu.

Migogoro kati ya walimu na wanafunzi inazuka shuleni Sayari pale ambapo mwalimu mkuu anapoenda kidato cha tatu kufundisha hesabu. Anagundua kuwa wanafunzi walikuwa na tabia ambazo hazikuwa za kawaida. Alipofanya msako na kupata mihadarati ndani ya dawati la mwanafunzi aitwaye Juma, anashikwa na hasira tele. Anaita mkutano wa dharura na walimu wake na wote kwa pamoja wanaamua kufanya msako madarasa yote na mabweni. Jambo hili linawakasirisha wanafunzi wa kidato cha tatu na wanaamua kuzua rabsha. Wanachoma madarasa mawili pamoja na ofisi ya naibu mkuu. Walimu walitoroka huku wasichana kwa wavulana wakitenda vitendo visivyokubalika 
hadharani. Walivunja milango na kubeba vitu vya dhamana na baadaye kutorokea mjini Bahati (uk.2024).

Juhudi za Inspekta Matano zilizaa matunda alipoweza kuwashika wahalifu hao na kuwatia mbaroni. Shule nyingi na watoto wengi wanaokolewa kutokana na maangamizi ya mihadarati. Kuwepo kwa vitengo vikuu serikalini kama vile Idara ya polisi, mahakama, idara ya magereza na vitengo vya utetezi wa haki za watoto huweza kutoa suluhu ya changamoto nyingi katika jamii kiuhalisia.

Migogoro kati ya walimu na wahalifu. Barua aliyoandika Makawe (Matata, Kamaliza na Wembe) inatoa taswira ya mgogoro kati ya mwalimu mkuu na wahalifu (uk.42). Kule kutishiwa maisha na wahalifu wasioogopa mkono wa sheria unaendeleza maudhui ya migogoro. Changamoto alizokumbana nazo mwalimu mkuu katika shule ya upili ya Sayari zilimpelekea kutafuta suluhisho. Aliungana na Inspekta Matano kuwasaka wahalifu hata ingawa alihatarisha maisha yake. Wananchi wanapaswa kuungana na askari ili kukomesha uhalifu katika jamii. Kuwepo kwa mahakama pia ni muhimu katika ukabilianaji wa changamoto za watoto. Wafungwa wanaweza kufanyiwa kesi mahakamani na pia waliodhulumiwa wanaweza kusaidiwa mahakamani kupata haki zao.

Sawa na kitabu cha Ahaa! Roda, upo mgogoro kati ya watu na nguvu zisizojulikana. Askari chini ya uongozi wa Inspekta Matano wanakabiliana na jinamizi la kifo kule Mapangoni. Kuuawa kwa Askari wawili George na Kabaka inaleta mgogoro kati ya Mwalimu mkuu na Inspekta. Mwalimu mkuu anaamini kuwa kifo kilitokana na mazingaombwe waliokasirika. Hata hivyo, Inspekta anaamini kuwa vifo vilisababishwa na wahalifu waliojificha mle ndani ya Mapango (uk.73). Ujasiri wa Inspekta matano ulisaidia kusuluhisha changamoto hii alipokabiliana na wahalifu hawa peupe. Watoto wana uwezo wa kukabiliana na changamoto zao kwa ujasiri bila uoga wa imani ya nguvu za mazingaombwe na ushirikina.

Mfamaji anaonyesha migogoro kati ya vizazi, utamaduni na elimu. Mapango yalikuwa mahali patakatifu ambapo wazee walitumia kuabudu. Baada ya uhuru, mambo yalibadilika. Vijana walichafua Mapangoni. Mihadarati ilifichwa huko na ubakaji ukaendelezwa. Pia Wazungu wenye elimu walipofika humo, walivuruga utamaduni huo wa kuabudu na wakatia Mapangoni najisi. Walitumia huko kuweka silaha na ukawa kitovu cha uhalifu. Mgogoro pia kati ya binadamu na nguvu zisizo za kawaida inashuhudiwa. Migogoro kati ya wahalifu na miungu inaonekana pale ambapo mzee Mfamaji anasema kuwa waliotia najisi mapangoni hufa mapema. Anatoa mfano wa Mzungu aliyeitwa Lot Massel mwenye elimu lakini hakufahamu utamaduni. Alipiga picha na kufanya utafiti mapangoni lakini alipokuwa akirudi kwao nyumbani, chombo cha usafiri kilizama baharini na wote wakafa (uk.59). Mfamaji anatoa suluhisho ya changamoto hizi kwa kutoa kafara kwa yeyote yule aliyemtembelea kwa nia ya kuzuru mapangoni. Alipitisha utamaduni kutoka kizazi kimoja hadi kingine. Walezi wanapaswa kuwaongoza, kuwaelekeza na kuwaelimisha watoto wao kwa njia inayokubalika katika jamii kwa mfano kuwaelimisha kuhusu neno la Mungu.

Kitabu cha Ahaa! Roda kimeangazia Elimu pia kama suluhisho ya kukabiliana na changamoto nyingi katika jamii. Watoto wa kijiji cha Tupe na Mabatini walipopata elimu, vijiji hivi vilibadilika. Usalama uliimarishwa, wazazi wakasaidika, kilimo kwa kutumia mbinu na teknolojia za kisasa zikaimarika, Umaskini ukaondoka na watu wakaishi katika vyumba vya kisasa na kuendesha magari makubwamakubwa (uk.92). Watoto wakifanya bidii shuleni, wataweza kubadilisha maisha yao na ya jamii kwa ujumla. Uhalifu na ukatili unaosababishwa na umaskini utasahaulika kabisa. Vitabu vya fasihi ya watoto, Ahaa! Roda na Mwisho wa Ujambazi vimetumia baadhi ya wahusika kukabiliana na changamoto za watoto.

Matokeo ya utafiti wa maktabani kuhusu jinsi changamoto za watoto zilivyokabiliwa kwenye vitabu vya fasihi teule vilioana na matokeo kutoka utafiti wa nyanjani kupitia majibu ya watafitiwa walipoulizwa iwapo waliweza kulinganisha hali katika vitabu teule walivyovisoma na changamoto halisi wanazopitia. Watoto asilimia hamsini $(N=10)$ walikubali kwamba waliweza kufananisha changamoto vitabuni na changamoto halisi wanazokumbana nazo. Hii inamaanisha kuwa watoto hawa wakisoma vitabu zaidi ya viwili bila shaka wataweza kupata changamoto kwenye hadithi watakazo fananisha na uhalisia wao. Ili kukamilisha 
shabaha hii ya pili, watoto waliotafitiwa waliulizwa kutoa maoni yao kuhusu vile changamoto vilivyokabiliwa vitabuni. Matokeo ya nyanjani yaliwasilishwa na kuchanganuliwa yafuatavyo;

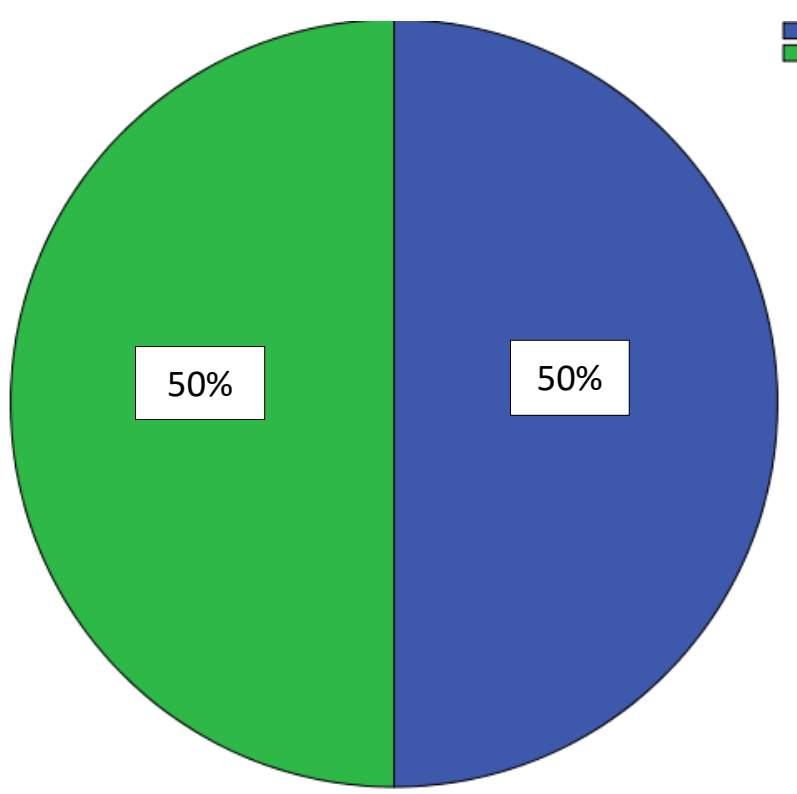

Matokeo yalionyesha kuwa walioweza kukutana na hali walizolinganisha na hali/ changamoto wanazopitia wenyewe walikuwa asilimia hamsini, $N=10$ na wale ambao hawakuweza kulinganisha hali vitabuni na hali zao binafsi walikuwa asilimia hamsini, $N=10$. Matokeo haya yanadhibitisha kuwa watoto wakisoma vitabu vingi zaidi wataweza kupata hali tofauti tofauti vitabuni ambazo wote wataweza kulinganisha na changamoto zao.

\section{Kulinganisha Hali Katika Vitabu Teule vya Fasihi ya Watoto na Changamoto Halisi.}

Watafitiwa waliulizwa iwapo walikutana na hali vitabuni Ahaa! Roda na Mwisho wa Ujambazi waliyoweza kulinganisha na changamoto walizopitia binafsi.

\section{Maoni ya Watoto Kuhusu Kukabiliana na Changamoto Vitabuni.}

Waandishi wa fasihi wakati mwingine huweza kuangazia suluhisho au ukabilianaji wa changamoto au migogoro vitabuni kupitia wahusika. Mtafiti aliwauliza sampuli lengwa iwapo walikubaliana na jinsi vitabu vya fasihi walivyovisoma vilikabiliana na changamoto. Watafitiwa waliweza kutoa maoni yao na matokeo yalibainishwa katika jedwali. Muundo wa skeli ya liketi ilitumiwa kukusanya majibu.

Jedwali 1: Maoni ya watafitiwa kuhusu kukabiliana na changamoto vitabuni.

\begin{tabular}{|c|c|c|c|c|c|}
\hline Kukabiliana na changamoto vitabuni & Nakubali & Sijaamua & & Sikubali & \\
\hline & $\%$ & $\mathbf{F}$ & $\%$ & $\mathbf{F}$ & $\%$ \\
\hline $\begin{array}{l}\text { Kupeleka wahalifu mahakamani } \\
\text { kuhukumiwa } \\
\text { mingi, kifungo } \\
\text { cha maisha au kifo }\end{array}$ & 17 & 0 & 0 & 3 & 15 \\
\hline $\begin{array}{l}\text { Kumfukuza/ kumpa talaka mama wa kambo } \\
\text { anayetesa watoto wasio wake }\end{array}$ & 70 & 3 & 15 & 3 & 15 \\
\hline
\end{tabular}

$119 \mid$ This work is licensed under a Creative Commons Attribution 4.0 International License. 
East African Journal of Swahili Studies, Volume 3, Issue 1, 2021

Article DOI: https://doi.org/10.37284/eajss.3.1.405

\begin{tabular}{|c|c|c|c|c|c|c|}
\hline \multirow{2}{*}{ Kukabiliana na changamoto vitabuni } & \multicolumn{2}{|c|}{ Nakubali } & \multirow{2}{*}{$\begin{array}{l}\text { Sijaamua } \\
\text { F }\end{array}$} & \multirow[b]{2}{*}{$\%$} & \multirow{2}{*}{$\begin{array}{l}\text { Sikubali } \\
\text { F }\end{array}$} & \multirow[b]{2}{*}{$\%$} \\
\hline & $\mathbf{F}$ & $\%$ & & & & \\
\hline $\begin{array}{l}\text { Kutetea haki za jinsia ya kike, kuwapa haki ya } \\
\text { kuridhi mali ya wazazi wao }\end{array}$ & 17 & 85 & 1 & 5 & 2 & 10 \\
\hline 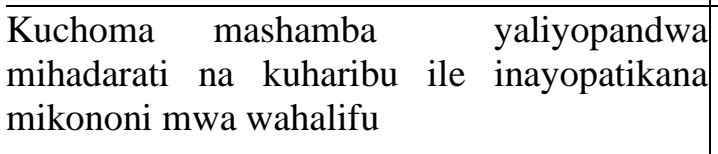 & 18 & 90 & 1 & 5 & 1 & 5 \\
\hline $\begin{array}{l}\text { Kuanzisha vyama vya kuwasaidia watoto } \\
\text { mayatima }\end{array}$ & 19 & 95 & 1 & 5 & 0 & 0 \\
\hline $\begin{array}{l}\text { Kuimarisha usalama vijijini, shuleni na popote } \\
\text { nchini }\end{array}$ & 20 & 100 & 0 & 0 & 0 & 0 \\
\hline $\begin{array}{l}\begin{array}{l}\text { Kuwaelimisha watoto katika taasisi } \\
\text { mbalimbali za elimu }\end{array} \\
\end{array}$ & i 19 & 95 & 0 & 0 & 1 & 5 \\
\hline
\end{tabular}

Jedwali laonyesha matokeo ya watoto kuhusu jinsi ya kukabiliana na changamoto. Asilimia tisini na tano $(N=95)$ walikubali kuwa, wahalifu wapelekwe mahakamani na kuhukumiwa kufungwa jela kwa miaka mingi, kifungo cha maisha au kifo ilihali asilimia tatu $(N=15)$ hawakukubali wahalifu wapelekwe mahakamani na kuhukumiwa kufungwa jela kwa miaka mingi, kifungo cha maisha au kifo. Hii ni kumaanisha kuwa watoto walikubali wahalifu wapelekwe mahakamani na kuhukumiwa kufungwa jela kwa miaka mingi, kifungo cha maisha au kifo.

Jedwali lili hili limeonyesha kuwa asilimia sabini $(N=14)$ ya watoto walikubali kufukuza au kumpa talaka mama wa kambo anayetesa watoto wasio wake, asilimia kumi na tano $(N=3)$ haikuweza kuamua kufukuza au kumpa talaka mama wa kambo anayetesa watoto wasio wake lakini asilimia kumi na tano $(N=3)$ haikukubali kufukuza au kumpa talaka mama wa kambo anayetesa watoto wasio wake. Hii ina maana kwamba watoto walikubali kufukuza au kumpa talaka mama wa kambo anayetesa watoto wasio wake kama namna ya kukabilianna na changamoto za maisha.

Asilimia themanini na tano $(N=17)$ ya watoto walikubali kutetea haki za jinsia ya kike; kuwapa haki ya kuridhi mali ya wazazi wao, nao asilimia tano $(N=1)$ haikutoa uamuzi kuhusu kutetea haki za jinsia ya kike; kuwapa haki ya kuridhi mali ya wazazi wao ilihali, asilimia kumi $(N=2)$ hawakukubali kutetea haki za jinsia ya kike; kuwapa haki ya kuridhi mali ya wazazi wao. Hii inamaanisha kuwa watoto walikubali kutetea haki za jinsia ya kike; kuwapa haki ya kuridhi mali ya wazazi wao kama mojawapo wa suluhu za changamoto wanazopitia.

Asilimia tisini $(N=18)$ ya watoto walikubali kuchoma mashamba yaliyopandwa mihadarati na kuharibu iliyopatikana mikononi mwa wahalifu, asilimia tano $(N=1)$ haikutoa uamuzi wa kuchoma au kutochoma mashamba yaliyopandwa mihadarati na kuharibu iliyopatikana mikononi mwa wahalifu, hata hivyo asilimia tano $(N=1)$ haikukubali kuchomwa kwa mashamba yaliyopandwa mihadarati na kuharibu iliyopatikana mikononi mwa wahalifu. Matokeo haya yanamaanisha kuwa, watoto walikubaliana na kuchoma kwa mashamba yaliyopandwa mihadarati na kuharibu iliyopatikana mikononi mwa wahalifu.

Asilimia tisini na tano $(N=19)$ ya watoto walikubali kuanzisha vyama vya kuwasaidia watoto mayatima, asilimia tano $(N=1)$ haikutoa uamuzi iwapo vyama vya kusaidia watoto mayatima vianzishwe au la. Hii inamaanisha kuwa watoto walikubali vyama vya kuwasaidia watoto mayatima vianzishwe ili kukabiliana na changamoto.

Jedwali laonyesha kuwa wanafunzi wote asilimia $(N=100)$ walikubali kuimarishwa kwa usalama vijijini, shuleni na popote nchini ili kukabiliana na changamoto.

$120 \mid$ This work is licensed under a Creative Commons Attribution 4.0 International License. 
Asilimia tisini na tano $(N=19)$ ya watoto walikubali kuelimisha watoto katika taasisi mbalimbali za elimu, nayo asilimia tano $(N=1)$ hawakukubali kuwaelimisha watoto katika taasisi mbalimbali za elimu. Matokeo haya yanadhihirisha kuwa watoto walikubali kuelimishwa kwa watoto katika taasisi mbalimbali za elimu.

\section{MATOKEO}

Baadhi ya matokeo yalikuwa kama yafuatavyo: Matokeo yaliyopatikana kutokana na uchanganuzi wa vitabu teule yalidhibitisha kuwepo kwa migogoro katika vitabu vya fasihi ya watoto. Aina za migogoro kama vile: mgogoro kati ya watoto na wazazi wao, mgogoro ndani ya mtoto mwenyewe, mgogoro kati ya mtoto na nguvu zisizo za kawaida na mgogoro kati ya mtoto na mazingira iliweza kubainika. Matokeo za kitakwimu pia zilidhibitisha kuwa watafitiwa walikubaliana na jinsi vitabu vilivyokabiliana na changamoto kama ifuatavyo:

Watoto walikubali wahalifu wapelekwe mahakamani na kuhukumiwa kufungwa jela, kwa miaka mingi, kifungo cha maisha au kifo. Watoto walikubali kumfukuza au kumpa talaka mama wa kambo anayewatesa watoto wasio wake kama namna ya kukabiliana na changamoto vitabuni. Watoto walikubali kutetea haki za jinsia ya kike kwa kuwapa haki ya kuridhi mali ya wazazi wao kama mojawapo ya suluhu za changamoto wanazopitia. Watoto walikubaliana na kuchoma kwa mashamba yaliyopandwa mihadarati na kuharibu iliyopatikana mikononi mwa wahalifu.

Hivyo basi vitabu vya fasihi ya watoto viliweza kutekeleza mahitaji ya watoto kwa kujenga hulka zao na kuwapa suluhisho ya changamoto zao. Watoto walikubaliana na jinsi vitabu vilivyokabiliana na changamoto.

\section{HITIMISHO NA MAPENDEKEZO}

K.I.C.D pamoja na wizara ya elimu wateue vitabu vya fasihi ya watoto vitakavyosomwa kwenye shule za msingi na ambavyo vyaweza kutekeleza mahitaji ya watoto humu nchini. Walimu wawe wakiteua vitabu vya fasihi na kuwapa wanafunzi wavisome. Waandishi wa vitabu waangazie maudhui tofauti yanayowafaa watoto wote wa kike na wa kiume. Walezi waweze kuwazoesha watoto wao kusoma vitabu vya fasihi ili kukuza maadili mema. Pia, Wasimamizi wa shule pamoja na walimu wahakikishe kuwa maktaba za shule zina vitabu vya fasihi ya watoto vya kutosha vilivyoteuliwa vizuri kulingana na maudhui mbalimbali yanayowakilishwa na wahusika. Walimu wasisitize usomaji wa vitabu hivi.

Mtafiti aliweza kupendekeza kuwa tafiti zingine zifanywe kuhusiana na utafiti huu lakini kuangazia zaidi vyombo vya habari. Kwamba hadithi zikiweza kuandikwa kwenye magazeti, kusomwa katika vipindi vya redio na runinga, kwenye vitabu vya kielektroniki, nyenzo mpya za usomaji zikizinduliwa kama vile simu tamba na 'Apple Ipads' na pia katika maendeleo ya kidijitali kama vile You Tube, Facebook na Twitter, Je fasihi hizi za watoto zaweza kuwanufaisha katika kutekeleza mahitaji yao?

Mtafiti pia aliweza kufanya utafiti wake katika shule moja. Anapendekeza kuwa tafiti zingine zifanywe katika shule nyingi maeneo tofauti tofauti nchini kwa wachochole na matajiri. Shule za upili na vyuo vikuu viweze kujumlishwa katika utafiti huu.

\section{MAREJELEO}

Alliance, UNICEF na WHO (2020). COVID-19: Kulinda Watoto Dhidi ya Dhuluma, Unyanyasaji na Kutelekezwa. https://www.mhinnovation.net/files/resources

Bakize, L. H. (2013). Changamoto Zinazoikabili Fasihi ya Watoto Tanzania Katika Kiswahili. Juz. 76 Dar es Salaam: Taasisi ya Taaluma za Kiswahili. Kur. 61-70.

Blake, F. (2020). Causes of Conflicts Between Parents and Teenagers. Wehavekids.com/parenting/sources-f-conflictsbetween-parents-and-Teenagers.

Creswell, J, (2008). Educational research: planning, conducting and evaluating quantitative and Qualitative research ( ${ }^{\text {rd }}$ ed.) Upper Saddle River, NJ: Pearson Education.

Creswell, J. W., \& Plano Clark, V. L. (2007). Designing and Conducting Mixed Methods Research. London: Sage Publications Ltd. 
Crippen, M, (2012). The Value of Children's Literature. Luther.edu/oneota-readingjournalarchaive/2012/the-value-of childrensliterature.

Dada, E. M. na Olaniyan, A. S (2020). The use of Literature as a tool for Holistic Development of Students Personality; U.K, UCRTD publishers.

Danley, B. (2014). Behaviorism Theory and its Relation to Instructional Design. (faculty, Mercer. Edu/codone- s/tco363/2014/behavioris m.pdf.)

Dickens, C. (2019). - https://tvmoon,ru/sw/astrolo giya-lyubvi-i-goroskopy/konfliktnye-situacii-vliterature-chto-takoe-konflikt-v.html

Gazeti La Taifa Leo, (2016, Novemba 21) Migogoro kati ya Wazee na vijana- SwahilihubTaifa Leo. - http://www:swahilihub.com/habari/ makala/migogoro- kati- ya- wazee- na vijana/13 102203459328.

Gazeti La Taifa Leo, (2020, Machi 17). Shina La Uhai; Ongezeko la mimba za mapema. Fumbo linalotatiza wataalamu.

Greene, J. C., Caracelli, V. J., \& Graham, W. F. (1989). Towards a Conceptual Framework Mixed Method Evaluation Designs. Educational Evaluation And Policy Analysis 11(3), 255-274. -http://dx.doi.org/10.3102/01623737011003255

Greenwood, J. (1988). Class Readers. New York: Oxford University Press.

Heather, M. \& Carmen, P. (2011). Gender Representation in a Selection of Children's Picture Books. A Skewed Ratio of Male to Female Characters? Undergraduate Research Journal for the Human Sciences (Online). Volume 10.

Hillman, J. (1974). An analysis of male and female roles in two periods of children's literature. The Journal of Educational Research, 68(2), 84-88.

Kadzin, A. E. (2001). Behaviour Modification in Applied Settings. (6 $6^{\text {th }}$ ed.). Wardsworth: Thomas Learning.
Kendra, C. (2019). History and key concepts of behavioural psychology. https://www.verywellmind.com>behavioralpsychology-4157183

Kostelny, K., Wessells, M., Chabeda-Barthe, J., na Ondoro, K. (2013). Learning about child in urban slums: A rapid ethnographic study in two urban slums in Mombasa of community-based child protection mechanisms and their linkage with the Kenya national child protection system. London: Interagency Learning Initiative on Community-Based Child Protection Mechanisms and Child Protection Systems.

Krebs, J. (2020). How to Write Compelling Conflicts: Create Conflicts in Stories. Master Class Staff.

Lacina, J. na Stetson, R. (2013). Young Children vol 68 ,publisher National Association for the Education of Young Children NAEYC, kur 3441.

LHRC (2017). Legal and Human Rights Centre; Siku ya mtoto wa Afrika: Changamoto zinazowakabili watoto Tanzania.

Lukov, V.A., Tyup, V.I., Propp, V.Y., Tamarchenko, N.D., Dickens, C. (2019). https://tvmoon,ru/sw/astrologiya-lyubvi-igoroskopy/konfliktnye-situacii-v-literaturechto-takoe-konflikt-v.html

Lyimo, E.B. (2014). "Usaguzi wa Kijinsia katika Vitabu vya Kiswahili vya Fasihi ya Watoto na Mtazamo wa Wadau nchini Tanzania." Tasnifu ya Uzamivu ya Chuo Kikuu cha Dar es Salaam.

Mapunjo, G. C. (2014). Usawiri wa Mwanamke Kama Kiongozi Katika Tamthiliya: Uchunguzi wa Kivuli Kinaishi na Nguzo Mama (Doctoral dissertation, The Open University of Tanzania).

Martinez, M. G. Yokota, J. na Temple, C. (2018). Thinking and Learning through children literature: John Hopkins University Press.

Matundura, E, Kobia, J. na M. Mukuthuria. (2013). Taswira Dumifu za Uana katika Fasihi ya Kiswahili ya Watoto, katika Mulika, Na. 32. Dar es Salaam: Taasisi ya Taaluma za Kiswahili. 
Mutuku, Y. (2017). Mwisho wa Ujambazi. Queenex Publishers Limited.

Ngugi, P. (2011): Language and Literacy Education: The State of Children's Literature in Kiswahili in Kenya. Berlin. Lambert Academic Publishing.

Ngugi, P. (2014). Fasihi ya Watoto katika Kutekeleza Mahitaji ya Kisaikolojia ya Mtoto, katika Kiswahili. Juz. 77. Dar es Salaam: Taasisi ya Taaluma za Kiswahili. 34

Ngugi, P. (2015). Mbinu na Mikakati ya Kutumia Fasihi ya Watoto kama Bibliotherapia katika Kioo cha Lugha. Juz. 13. Dar es Salaam: Taasisi ya Taaluma za Kiswahili.

Njogu, K. na Chimera, M. (1999) Ufundishaji wa Fasihi: nadharia na mbinu. Nairobi. Jomo Kenyatta Foundation.

Nyambeka, T. (2017) Ahaa! Roda. Queenex Publishers Limited.

Odaga, A. B. (1974). —-Literature for Children and Young People, Tasnifu ya B.A. Chuo Kikuu cha Nairobi. (Haijachapishwa).

Porter, A. (2008). The Cambridge Introduction to Narrative: The Cambridge Introduction to Literature. Cambridge University Press.

Rokkit, (2019). Hali ya migogoro kati ya watoto wa shule na uamuzi wao. Migogoro ya darasa; Aina, uamuzi, kuzuia.- Rokkit.ru/sw/pricheski/konflik tnye-situacii-mezhdu-shkolnikami-i-ihreshenie/

Salehi, A. (2011). Foundations and Principles of Psychological Content of Children's Books. Makala yaliyowasilishwa katika Kongamano la Fasihi ya watoto. Bongokok.-Psrcentre.org./ images/extraimages/36pdf. 27-2-2014.

Senkoro, E. M. K. (1987). Fasihi na Jamii. Dar es salaam: Press and Publicity Centre.

Thompson, R. A., Goodman, M. na Waters, S. F. (2018). Parent Child Conflict. Davis, CA, USA. University of California.
Wamitila, K. W. (2003). Kamusi Ya Fasihi, Istilahi na Nadharia.Nairobi: Focus Books.

Wamitila, K.W. (2008). Misingi ya Uchunguzi wa Fasihi. Nairobi. Vide - Muwa Publishers Ltd.

Wanjala F. S, (2020). Safina Ya Utafiti wa Fasihi Simulizi. Elgon Epitome Publishers Ltd.

Wanjala, F. S. (2011). Kitovu cha Fasihi Simulizi. Tanzania. Serengeti Educational Publishers.

Wasilwa, S. (2021). Kenya: NACADA-Watoto Wadogo Wanatumia Dawa za Kulevya.dw.com/sw/Kenya-nacada-yasema-watotowadogo-wanatumia-dawa-za-kulevya/a56358501

123 This work is licensed under a Creative Commons Attribution 4.0 International License. 